\title{
CHARACTERIZATION OF MECHANICAL PROPERTIES IN WELD METAL USING INVERSE MODELLING
}

\author{
J. Beddeleem ${ }^{1}$, W. De Waele ${ }^{1}$, S. Hertelé ${ }^{1}$, M. Verstraete ${ }^{1}$ and K. Van Minnebruggen ${ }^{1}$ \\ ${ }^{1}$ Ghent University, Laboratory Soete, Belgium
}

\begin{abstract}
:
Nowadays, more oil and gas transportation pipelines are constructed in areas with permafrost and/or higher seismic activity. These pipelines can be subjected to longitudinal plastic deformations necessitating a strain based design. Since girth- and seam welds are critical in terms of structural integrity, it is desirable to know their mechanical properties. In a strain based design context, the accurate determination of yield strength and hardening are necessary. A longitudinally extracted (is parallel to the pipe axis) specimen notched at the weld region and loaded in tension, in combination with inverse modelling is assumed to be a valuable tool to determine these properties. This notched cross weld test ensures that the largest deformations will occur at the weld, thereby allowing to fully determine the stress-strain behaviour of the weld metal. Inverse modelling combines experimental full-field strain data with numerical simulations to determine the constitutive parameters. Strains will be measured experimentally and compared with simulated data. By minimizing their difference, i.e. a certain cost function, a correspondence is found and the desired parameters are determined. This paper focuses on one aspect of the inverse modelling framework, the development of the parametric finite element model.
\end{abstract}

Keywords: mechanical properties, inverse modelling, notched tensile test, finite element analysis.

\section{INTRODUCTION}

Due to the ever-increasing consumption of energy and the fact that fossil fuels are exhaustible, an increasing number of pipelines is constructed in more hostile environments (e.g. permafrost, prone to landslides...). Due to these extreme conditions, the pipelines can be subjected to plastic deformations in the longitudinal direction. Since welds are critical sections of a pipeline [1], it is important in a strain based design context to accurately know their mechanical properties such as yield strength and hardening behaviour.

\section{METHODS TO ACHIEVE MECHANICAL PROPERTIES OF WELD METAL}

\subsection{Tensile test on round bar specimen}

Several methods exist to determine the mechanical properties of the weld metal. At first, a round bar sample can be taken from the weld in the circumferential direction. In [2] and [3], specimens were machined from three different positions in the weld cross section (see Figure 1). This allows to determine the change of the weld metal properties over the height. The first position is close to the weld cap, position two is at mid thickness and position three is close to the weld root. The limitations of this method are:

- The obtained properties represent the circumferential direction of the weld, which might be different from those in the longitudinal direction due to anisotropy. It is possible to take a sample in the longitudinal direction, however this sample will most likely neck at the base metal, because the base metal usually has a lower strength than the weld metal.

- The obtained properties strongly depend on the sample position over the height of the weld and do not represent full thickness properties (Figure 2).

Despite its drawbacks, this specimen is almost unanimously used in strain based design to determine the mechanical properties of weld metal, because it is straightforward to implement. 


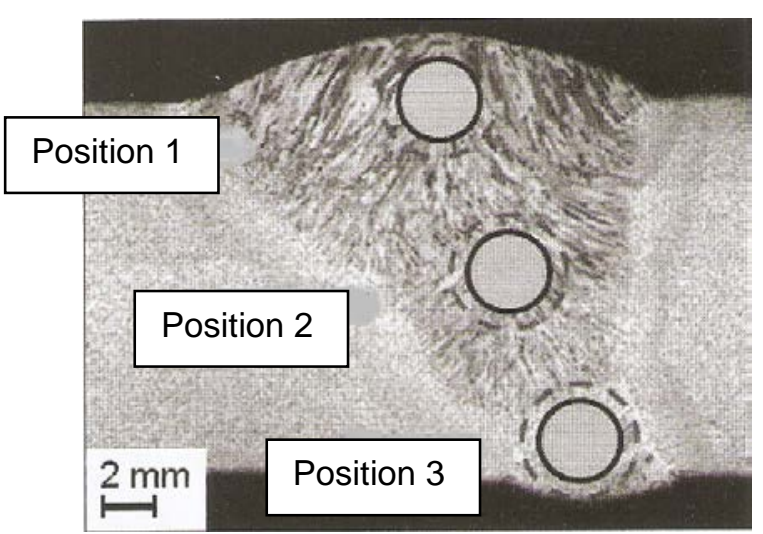

Figure 1. Sketch of the three positions of the round bar test specimen [2].

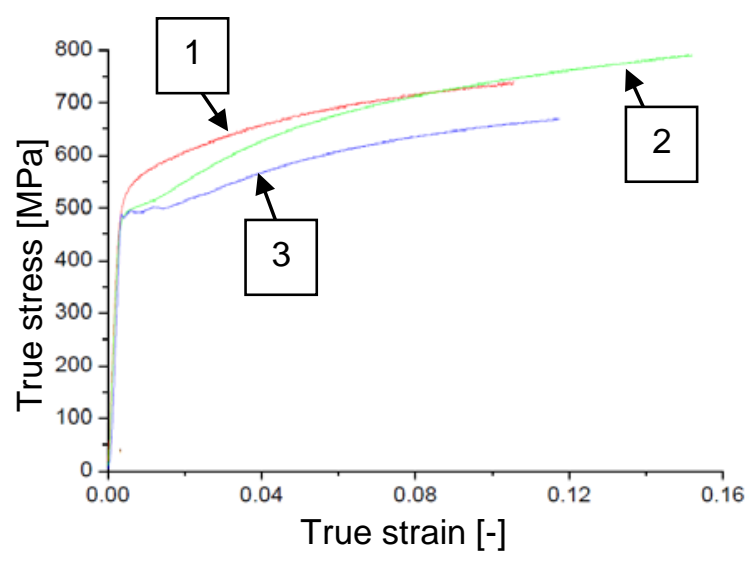

Figure 2. Stress-strain curves are dependent of sample position [3]

\subsection{Hardness measurements}

By making use of hardness measurements the tensile strength of a metal can be estimated [4]. The tensile strength (expressed in MPa) of steel is approximately 3.2 times the Vickers hardness (expressed in HV10) [5].

The disadvantage of this method is that only the tensile strength is determined. On the other hand, it is possible to determine the variation of tensile strength between the base metal, HAZ and the weld metal both, in the height and longitudinal direction.

\subsection{Notched tensile test combined with inverse modelling}

The next method includes a tensile test on a notched specimen in combination with inverse modelling. This method enables to determine full-range longitudinal stress-strain properties for the weld metal. Key to this ability is that it can be used in the presence of heterogeneous strain fields.

By using a notched sample, the largest deformations occur in the region of the notch and it is clear that the sample will eventually neck in this region if the local area reduction has adequately been chosen. This contrasts a prismatic tensile specimen, where the fracture will occur at the lowest strength region. Reported in literature as a suited specimen geometry is a notched specimen with an area reduction of $60 \%$ at the notch (Figure 3) [3]. This ratio is important to ensure that the plastic deformations mainly occur in the weld.

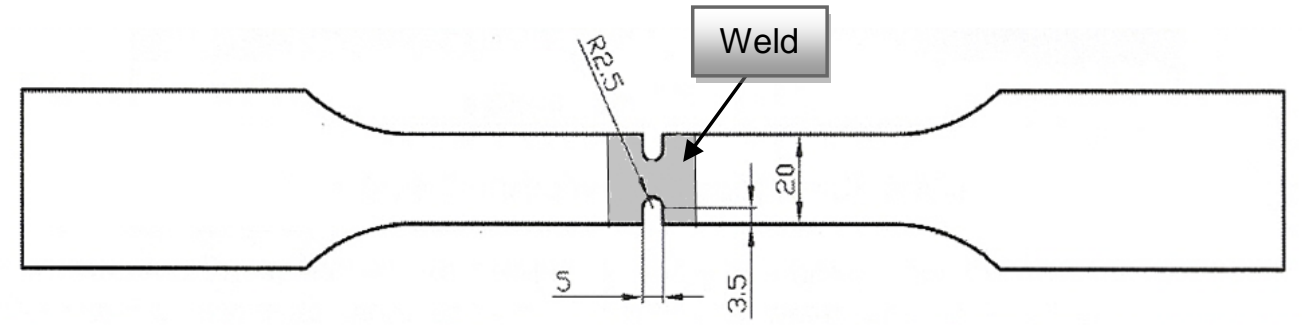

Figure 3. A notched tensile test specimen [3]

The inverse modelling framework is schematically summarized in Figure 4. The strains in the longitudinal direction, in close vicinity to the notch, are measured experimentally and compared with results of a finite element model that represents the same configuration but has estimated weld metal properties. The experimental strain measurement is performed using digital image correlation, an optical measuring technique which monitors surface deformations and derives strains by differentiation. To apply this technique, a random speckle pattern is applied and the movements of these speckles are monitored during the experiment [6-8].

At different times during the tensile test, the difference between the measured and numerically calculated strains is quantified using a cost function. When this function is minimal, the input weld metal properties are considered to yield the closest possible representation of the experimental ones. There are a lot of algorithms available to minimize this function, a common by used one is Nelder-Mead [9-11]. This algorithm is highly straightforward to program, for instance compared with Gauss-Newton [12]. On the other hand, the latter has been found more efficiently in terms of iterations $[13,14]$. A disadvantage of most algorithms, including Nelder-Mead, is that the algorithm can converge to a local minimum. To avoid this, computationally intensive algorithms have to be applied, such as genetic algorithms $[15,16]$. 


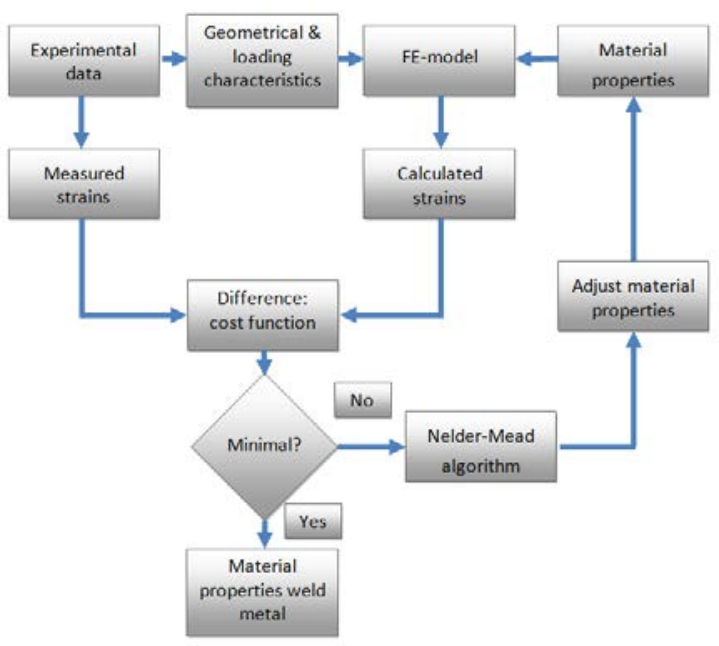

Figure 4. Inverse modelling

\subsection{Local strength determination methods}

Two other methods which can be used to evaluate the local strength of girth welds are instrumental hardness and micro tensile specimens [17]. The first method performs hardness measurements in many different positions to have a clear picture of the hardness of the specimen. The second method, tensile tests are performed on very small specimens, which have a thickness of $0.5 \mathrm{~mm}$ and a width of $2 \mathrm{~mm}$.

\section{THE FINITE ELEMENT MODEL}

A fully parametric finite element model is used to simulate the cross weld notched tensile test. The geometrical model represents a half test specimen by imposing a longitudinally oriented symmetry plane (Figure 5).

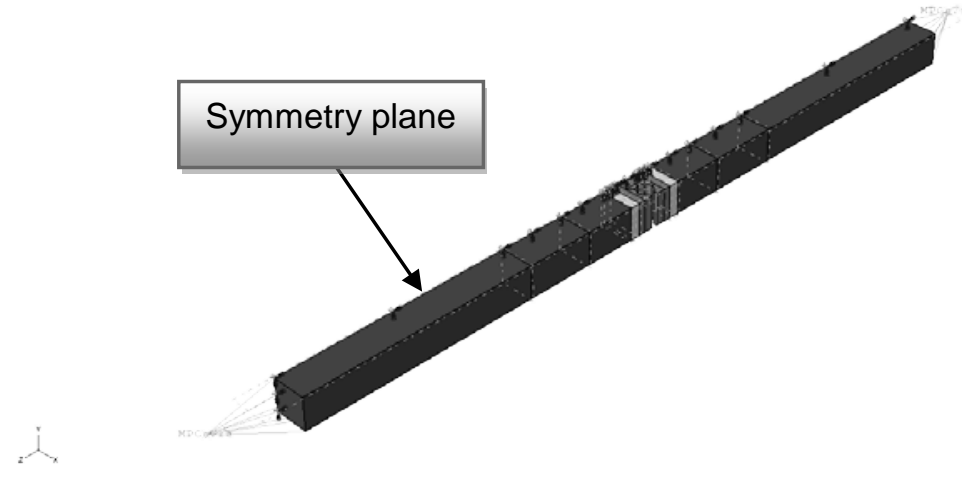

Figure 5. The finite element model

Two types of notch geometries can be indentified representing possible preparations during machining. These are the milled U-notch and the drilled keyhole notch (see Figure 6).
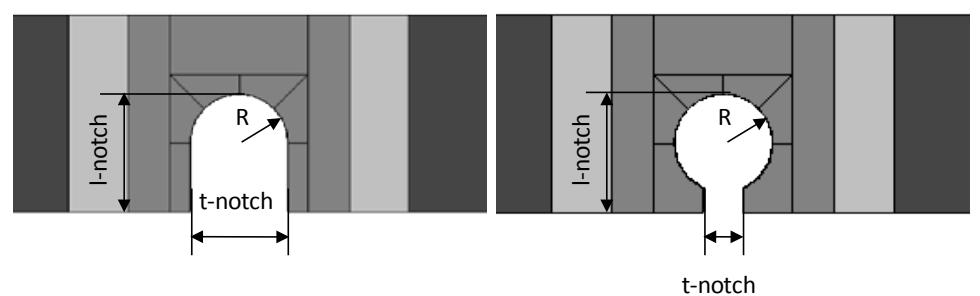

Figure 6. A U-notch (left) and a keyhole notch (right) with material zones, from left to right: base metal, heat-affected zone, weld metal, heat-affected zone, base metal

The heat affected zone directly adjacent to the weld, is characterized by different microstructures in comparison with the weld and base metal. To model this pragmatically, the test specimen is divided in 
partitions with different material properties. The different zones are indicated in Figure 6. The used material model is Ramberg-Osgood (Figure 7), where the yield strength and the strain hardening exponent are the parameters describing post-yield behaviour. The hardening exponent determines the increase of stress as a function of increasing plastic deformation.

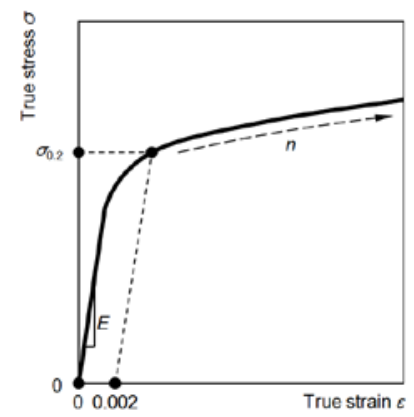

Figure 7. The material model according to Ramberg-Osgood

The curve of Figure 7 can be represented by (1) [18]:

$\varepsilon=$ true strain $[-]$

$$
\varepsilon=\frac{\sigma}{E}+0.002\left(\frac{\sigma}{\sigma_{0}}\right)^{n}
$$

$\sigma=$ true stress [MPa]

$E=$ Young modulus [Mpa]

$\sigma_{0}=$ yield strength $(0.2 \%$ proof stress $)$ [MPa]

$n=$ strain hardening exponent [-]

One end of the piece is clamped and at the other end a longitudinal displacement is imposed. Therefore, multipoint constraints are used. With this type of constraint, the load is applied at one point which is connected to the complete end surface of the piece. Knowledge of this load is important, because the difference between the experimentally and numerically determined strains is calculated at equal load values.

As one of the prerequisites to obtain a desired degree of accuracy, it is necessary that the specimen is meshed into a sufficient number of elements. This can be achieved by deliberately imposing the edge seeds. A higher number of elements should result in more accurate calculations, but requires more time to calculate. The number of nodes on an edge is based upon a reference seed density, which is multiplied by a mesh factor. As such, the reference mesh corresponds with a mesh factor one, a finer mesh with a mesh factor greater than one and vice versa. As for the element type, 20-node quadratic continuum bricks with a reduced integration have been used.

To use inverse modelling, it is necessary that the strains in the longitudinal direction (Z-direction, Figure 8) can be automatically transferred to an output file. In this model, a path is described on the symmetry plane of the upper surface along which the longitudinal strains are extracted.

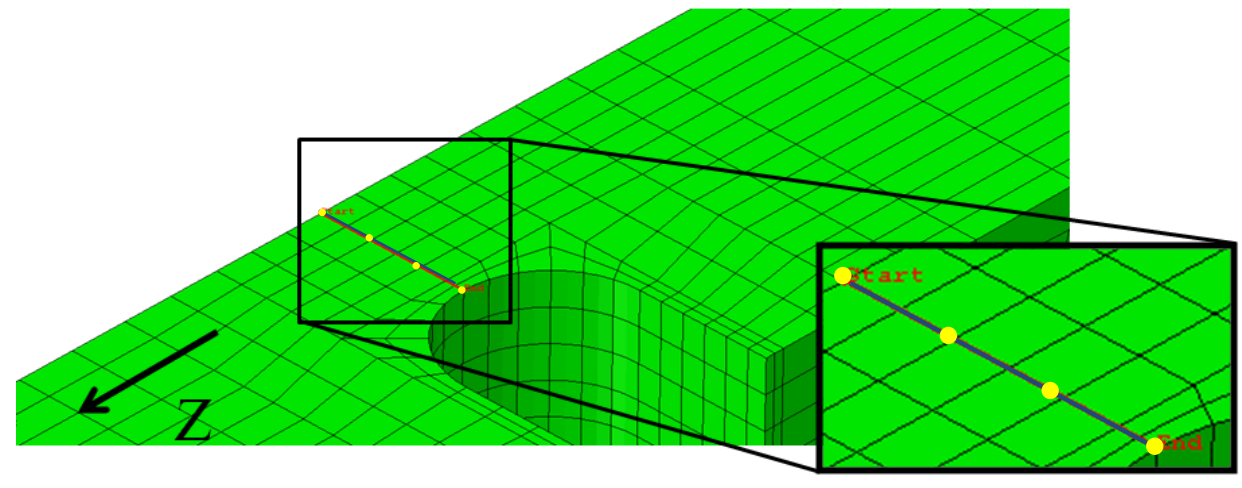

Figure 8. The path on the symmetry line of the upper surface 
To validate the developed finite element model, a thin plate with a hole has been simulated, because it has a well-known analytical solution. To this purpose, additional symmetry conditions were added in Figure 9. When the plate is remotely loaded with a constant stress in the longitudinal direction, the stress at the edge of the hole is three times larger than the remote stress (see Figure 9) [19].

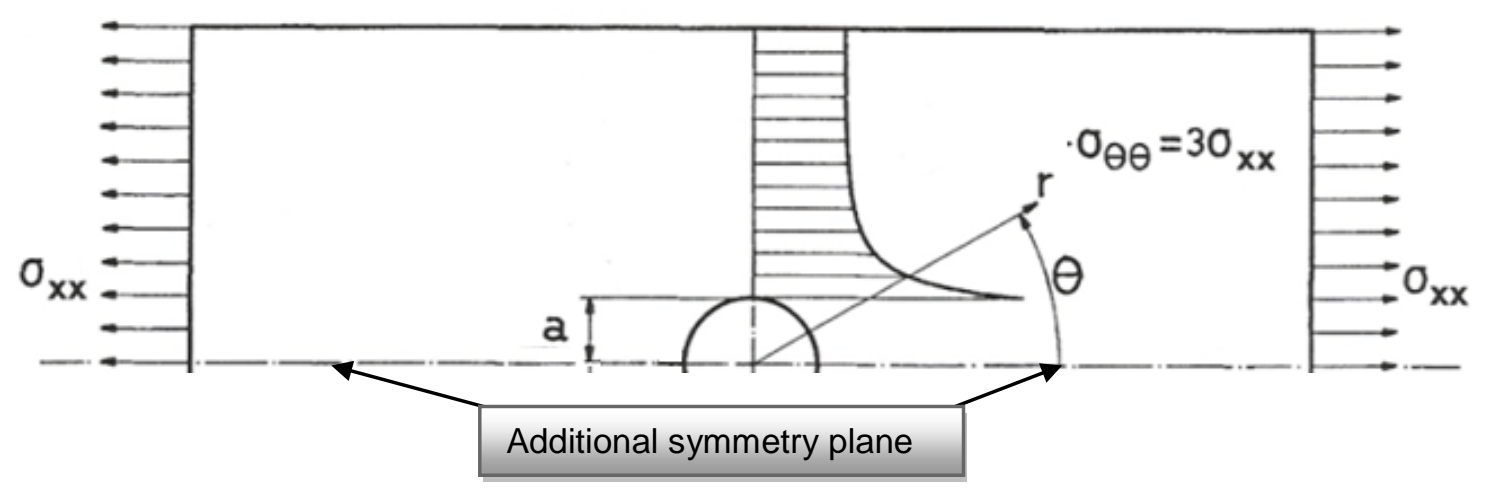

Figure 9. The analytical solution of the simulated stress concentration [19]

In the finite element model, the stresses were calculated at different points on the symmetry plane at the upper surface, this is the same path of Figure 8. To obtain stress concentrations, these stresses have been divided by the remote stress, which has been calculated by dividing the applied force by the cross section of the plate. Figure 10 plots the evolution of stress concentration as a function of the distance from the notch, for different mesh factors. It can be concluded that the regression of the different lines are almost the same. Only near the tip the result is marginally different. However, the correspondence with the theoretical solution is satisfying.

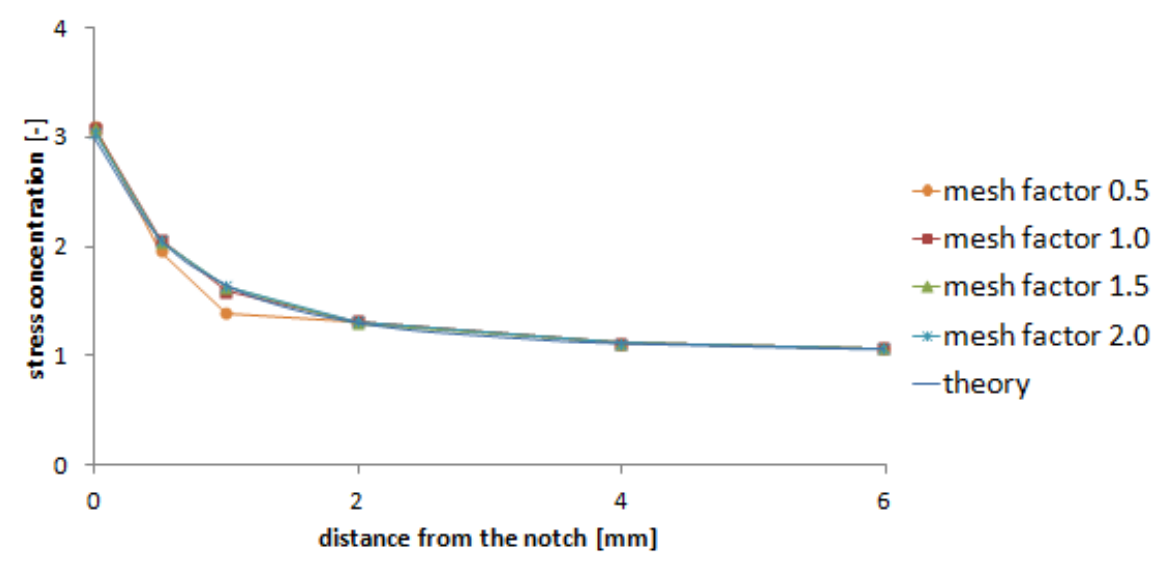

Figure 10. The evolution of the stress concentration for different mesh factors

In Table 1, a comparison is made between the different mesh factors in terms of number of elements, computation time and the maximum percentual deviation from the theoretical values. From this table, it can be concluded that a mesh factor of one is sufficient.

Table 1. Comparison of the different mesh factors

\begin{tabular}{|c|c|c|c|}
\hline Mesh factor & $\begin{array}{c}\text { Number of } \\
\text { elements }\end{array}$ & $\begin{array}{c}\text { Computation } \\
\text { time [s] }\end{array}$ & $\begin{array}{c}\text { Max. deviation } \\
{[\%]}\end{array}$ \\
\hline 0.5 & 704 & 39 & 15.41 \\
\hline 1.0 & 2116 & 85 & 3.47 \\
\hline 1.5 & 3844 & 141 & 2.64 \\
\hline 2.0 & 6386 & 216 & 1.66 \\
\hline
\end{tabular}


Inverse modelling is assumed to offer unmistakable advantages for the determination of the longitudinal mechanical properties of girth weld metal. In this paper, a finite element model is discussed, which constitutes a first building block of an inverse modelling framework. This finite element model is fully parametric and from an analytical validation, a suited mesh density was derived to obtain numerically accurate calculations. This model will contribute to a better understanding on how to obtain longitudinal girth weld properties in a strain-based context.

\section{REFERENCES}

1. R. M. Denys, Weld metal strength mismatch: past, present and future

2. A. Fonzo, G. Mannucci, L. F. Di Vito, G. Richard, Advanced tensile behaviour evaluation of girth welds, in ISOPE-2008, 2008. p. 7

3. A. Fonzo, L. F. Di Vito, Joined use of laboratory testing and finite element analysis for evaluating the load bearing capability of double joint girth welds. 2007.

4. $\quad$ Specification for welding of steel pipelines on land and offshore in British standard BS 4515:1995.

5. NBN EN ISO 18265, Metalen - Conversie van hardheidswaarden, 2003.

6. M. A. Sutton, J.J. Orteu, H. W. Schreier, Image Correlation for shape,motion and deformation measurements, basic concepts, theory and applications. 2009: Springer. 321 pages.

7. D. Lecompte, et al., Quality assessment of speckle patterns for digital image correlation. Optics and Lasers in Engineering, 2006. 44(11): p. 1132-1145.

8. K. De Keyser, F.Van Acker, S. Hertelé, M. Verstraete, W. De Waele, R. Denys, Validation of a wide plate fintie element model using digital image correlation, in Sustainable Construction and Design 2011 (SCAD) 2011: Ghent. p. 416-423.

9. J.A.Nelder and R. R. Mead, A simplex-method for function minimization Computer Journal, 1965. 7(4): p. 308-313.

10. J.C. Lagarias, et al., Convergence properties of the Nelder-Mead simplex method in low dimensions. Siam Journal on Optimization, 1998. 9(1): p. 112-147.

11. J.Kajberg and G.Lindkvist, Characterisation of materials subjected to large strains by inverse modelling based on in-plane displacement fields. International Journal of Solids and Structures, 2004. 41(13): p. 3439-3459.

12. S. Cooreman, et al., Identification of mechanical material behavior through inverse modeling and DIC. Experimental Mechanics, 2008. 48(4): p. 421-433.

13. fminsearch, Computes the unconstrained minimimum of given function with the Nelder-Mead algorithm. [cited 201110 December]; Available from:

http://help.scilab.org/docs/5.3.2/en US/fminsearch.html.

14. J. E. Dennis, R. B. Schnabel., Numerical Methods for Unconstrained Optimization and Nonlinear Equations 1983. Reprinted as Classics in Applied Mathematics 16, SIAM,Philadelphia, Pennsylvania, USA, 1996.

15. K. A. Hummel, J. P.G. Sterbenz, self-organizing systems: third international workshop. 2008. 240 pages.

16. B. Reusch, Computational intelligence: theory and applications. 2001: Springer. 1003 pages.

17. W. C. Mohr. Local strength testing for girth welds in X-100 pipes in Internationall Offshore and Polar Engineering Conference 2007. Lisbon

18. W. Ramberg, W. Osgood. Descreption of stress-strain curves by three parameters. 1943 [cited 201111 December ]; Available from: http://www.apesolutions.com/spd/public/NACA-TN902.pdf.

19. W. Van Paepegem, W. De Waele, Mechanica van materialen, course 2nd bachelor in Engineering, Ghent University, 2011. 